\title{
Measurement of residual stress in multicrystalline silicon ribbons by a self-calibrating infrared photoelastic method
}

\author{
M. C. Brito, ${ }^{\text {a) }}$ J. P. Pereira, J. Maia Alves, J. M. Serra, and A. M. Vallera \\ Faculdade de Ciências da Universidade de Lisboa, Departamento de Física/CFMC, \\ Lisboa, 1749-016 Portugal
}

(Received 13 May 2004; accepted 4 October 2004; published online 17 December 2004)

\begin{abstract}
This article reports on a method for the measurement of residual stress in multicrystalline silicon ribbons, based on the infrared photoelastic technique. This self-calibrating method allows the in situ determination of the photoelastic coefficients and can thus be used for any crystal orientation. The method was validated by the experimental determination of the photoelastic coefficient of monocrystalline (100) silicon wafers and by comparison with strain measurements using asymmetrical x-ray diffraction. The distribution of residual stress in multicrystalline silicon ribbons was also measured. The results showed strong evidence for tensile stress in the central region and compressive stress near the edges of the ribbons. Both the measured residual stress and the photoelastic coefficient distributions are correlated to grain boundaries. (C) 2005 American Institute of Physics. [DOI: 10.1063/1.1823654]
\end{abstract}

\section{INTRODUCTION}

Residual stress is a limiting factor in the production of multicrystalline silicon ribbons for photovoltaic applications, in particular for processes using perpendicular growth from the liquid such as edge defined growth ribbons (EFG), ${ }^{1}$ silicon sheet from powder (SSP), ${ }^{2} \mathrm{~S}$ ribbon, ${ }^{3}$ or those being studied in our laboratory based on growth from a molten zone. ${ }^{4}$ Furthermore, stress is strongly correlated with the formation of electrically active defects that can severely undermine the efficiency of the solar cells. ${ }^{5,6}$

Photoelasticity is a well known nondestructive method to study stress. Since silicon is opaque in visible light but transparent in the near infrared, the infrared photoelastic method has been used to study the residual stress in silicon wafers. ${ }^{7-10}$ In order to increase the sensitivity of the measurement, a phase-stepping technique may be used. ${ }^{11,12}$ However, the application of the photoelastic method requires the knowledge of the photoelastic coefficient, a material constant that depends on the crystal orientation. ${ }^{13,14}$ This is a problem for multicrystalline silicon ribbons where the orientation of the individual crystalline grains is, in general, not known. In this article we present a self-calibrating infrared photoelastic method that allows the in situ determination of the photoelastic coefficients and can therefore be used to measure residual stress in multicrystalline ribbons with arbitrary grain orientation.

\section{PHOTOELASTIC METHOD}

For a thin, transparent and isotropic material the principal components of the refraction index are coincident with the principal components of the stress and can be related by the stress-optic law

\footnotetext{
${ }^{\text {a) }}$ Author to whom correspondence should be addressed; electronic mail: mcbrito@fc.ul.pt
}

$$
n_{1}-n_{2}=C\left(\sigma_{1}-\sigma_{2}\right)
$$

where $n_{1}$ and $n_{2}$ are the principal components of the refraction index, $\sigma_{1}$ and $\sigma_{2}$ the principal components of the stress tensor and $C$ is a constant, called stress-optic constant or photoelastic coefficient.

When light propagates in the material, the change of refractive indices, due to the residual stress in the sample, introduces a phase difference, $\delta$, between the two electrical field components of the light. It can be shown ${ }^{9}$ that this phase difference, also known as the isocromatic parameter or fractional order, can be related to the difference of the principal components of the stress tensor by

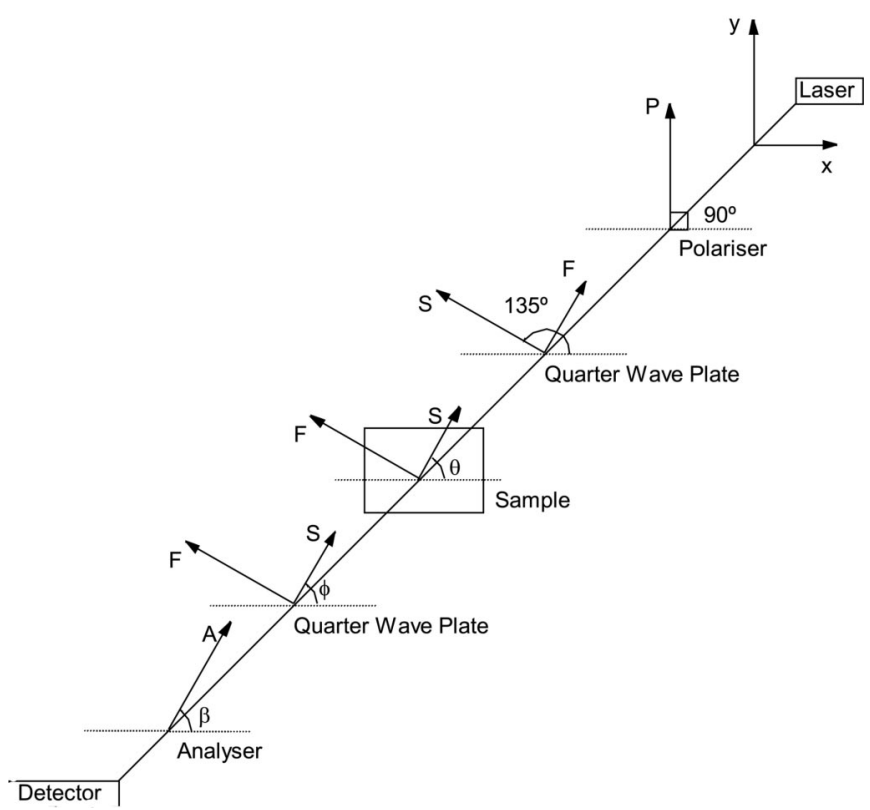

FIG. 1. Optical arrangement for phase shifting photoelastic measurement of stress. 


$$
\delta=\frac{2 \pi}{\lambda} d C\left(\sigma_{1}-\sigma_{2}\right)
$$

where $d$ is the tickness of the sample and $\lambda$ the radiation wavelength. If the photoelastic coefficient $C$ is known, the difference of the principal components of the stress tensor can thus be determined by measuring the phase difference $\delta$.

The phase difference $\delta$ can be determined using the phase shifting photoelastic method. ${ }^{11,12}$ Figure 1 shows the setup configuration. It includes (i) the light source (laser) followed by (ii) a linear polarizer and (iii) a quarter wave plate, with a $45^{\circ}$ rotation in order to produce circularly polarized light; (iv) the sample, characterized by the stressinduced isocromatic phase difference $\delta$ and the isoclinic angle $\theta_{n}$ (the angle between the principal refractive indice direction and the reference axis); (v) a second quarter wave plate, with orientation $\phi$; (vi) a second linear polarizer, called the analyzer, with orientation $\beta$ and $(v)$ the detector.

For this optical arrangement, the components of the light polarization vector along the analyzer axis, $U$, and perpendicular to the analyzer axis, $V$, can be obtained using Jones' calculus $^{15}$

$$
\begin{aligned}
{\left[\begin{array}{l}
U \\
V
\end{array}\right]=} & {\left[\begin{array}{cc}
\cos \beta & \sin \beta \\
-\sin \beta & \cos \beta
\end{array}\right]\left[\begin{array}{cc}
1-i \cos 2 \phi & -i \sin 2 \phi \\
-i \sin 2 \phi & 1+i \cos 2 \phi
\end{array}\right]\left[\begin{array}{cc}
\cos \frac{\delta}{2}-i \sin \frac{\delta}{2} \cos 2 \theta_{n} & -i \sin \frac{\delta}{2} \sin 2 \theta_{n} \\
-i \sin \frac{\delta}{2} \sin 2 \theta_{n} & \cos \frac{\Delta}{2}-i \sin \frac{\delta}{2} \cos 2 \theta_{n}
\end{array}\right]\left[\begin{array}{ll}
1 & i \\
i & 1
\end{array}\right] } \\
& \times\left[\begin{array}{l}
0 \\
1
\end{array}\right] E_{0} e^{i \omega t},
\end{aligned}
$$

where each matrix represents the rotation and retardation introduced by each optical element, and $E_{0} e^{i \omega t}$ is the incident light vector.

The detected intensity of the light is proportional to the square of the amplitude. Multiplying the matrices of Eq. (3) and adding a term, $I_{0}$, to represent background/stray light illumination, we obtain the intensity of the light transmitted for arbitrary positions of the second quarter wave plate and analyzer

$$
\begin{aligned}
I= & I_{0}+I_{m}[\sin (2(\varphi-\phi)) \cos \delta]+I_{m}[\cos (2(\varphi \\
& \left.-\phi)) \sin \left(2\left(\phi-\theta_{n}\right)\right) \sin \delta\right] .
\end{aligned}
$$

A set of different configurations of the second quarter wave plate and of the analyzer can be judiciously chosen in order to extract the isoclinic angle, $\theta_{n}$ and the phase difference $\delta$. Table I summarizes the intensities obtained from Eq. (6) for six such configurations. Combining and simplifying these equations we obtain, for the isoclinic angle, $\theta_{n}$ :

$$
\theta_{n}=\frac{1}{2} \arctan \left(\frac{I_{5}-I_{3}}{I_{4}-I_{6}}\right)
$$

and for the phase difference $\delta$ :

$$
\begin{aligned}
& \delta=\arctan \left(\frac{I_{5}-I_{3}}{\left(I_{1}-I_{2}\right) \sin 2 \theta_{n}}\right), \\
& =\arctan \left(\frac{I_{4}-I_{6}}{\left(I_{1}-I_{2}\right) \cos 2 \theta_{n}}\right) .
\end{aligned}
$$

These results, combined with Eq. (2), show that the optical arrangement shown in Fig. 1 can be used for the measurement of residual stress in transparent and isotropic materials, if the photoelastic coefficient $C$ is known.

\section{PHOTOELASTIC METHOD APPLIED TO SILICON RIBBONS}

Unlike isotropic materials, in a crystal the direction of the principal components of the stress tensor are not, in general, coincident with those of the refraction indices and the photoelastic coefficient $C$ is not constant but depends on the orientation of the crystal. ${ }^{13}$ Liang and $\mathrm{Pan}^{14}$ have shown that the photoelastic coefficient for (100) silicon wafers is in the range $(1.87-2.44) \times 10^{-11} \mathrm{~Pa}^{-1}$ and is explicitily given by

$$
C=\left(1.87+0.57 \sin ^{2} 2 \theta\right) 10^{-11} \mathrm{~Pa}^{-1},
$$

where $\theta$ is the angle defining the direction of the principal components of the stress tensor with respect to the laboratory reference frame. This angle is related to the principal orientations of the refractive indices (i.e., the measured isoclinic parameter, $\theta_{n}$ ) by the trascendental equation ${ }^{12}$

$$
\tan 2\left(\theta_{n}-\theta\right)=\frac{8.97 \sin 4 \theta}{12.22 \sin ^{2} 2 \theta+3.25 \cos ^{2} 2 \theta} .
$$

For multicrystalline silicon it is not practical to determine the crystal orientation of all the grains. However, the photoelastic coefficient can be estimated at every measured point of

TABLE I. Different configurations used to determine the isocromatic parameter $\delta$.

\begin{tabular}{lcc}
\hline \hline$\phi$ & $\beta$ & Output \\
\hline 0 & $\pi / 4$ & $I_{1}=I_{0}+I_{m} \cos \delta$ \\
0 & $3 \pi / 4$ & $I_{2}=I_{0}-I_{m} \cos \delta$ \\
0 & 0 & $I_{3}=I_{0}-I_{m} \sin 2 \theta_{n} \sin \delta$ \\
$\pi / 4$ & $\pi / 4$ & $I_{4}=I_{0}+I_{m} \cos 2 \theta_{n} \sin \delta$ \\
$\pi / 2$ & $\pi / 2$ & $I_{5}=I_{0}+I_{m} \sin 2 \theta_{n} \sin \delta$ \\
$3 \pi / 4$ & $3 \pi / 4$ & $I_{6}=I_{0}-I_{m} \cos 2 \theta_{n} \sin \delta$ \\
\hline \hline
\end{tabular}




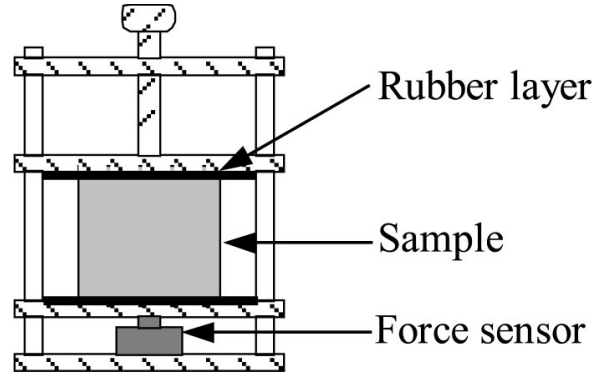

FIG. 2. Setup for measurement of the applied stress.

the sample by applying different (and known) external stresses and measuring the total stress. The residual stress at any point of the sample can thus be computed by extrapolation of the curve applied stress versus measured stress to zero applied stress.

\section{EXPERIMENTAL SETUP}

The optical arrangement we used is the one described schematically in Fig. 1. The light source is a $10 \mathrm{~mW}$, $1310 \mathrm{~nm}$ infrared laser diode Mitsubishi ML725B8F electronically modulated at a frequency of $20 \mathrm{kHz}$. A HeNe laser was also installed for alignment purposes. The light detector is a high speed InGaAs detector, DET410, from Thorlabs with an 800-1800 nm range. The total range of the $X Y$ translation scanning system is $10 \times 10 \mathrm{~cm}^{2}$ and the maximum resolution is $25 \times 25 \mu \mathrm{m}^{2}$, well bellow the $1 \mathrm{~mm}$ laser spot size. The detector output signal is measured by a Stanford RF850 lock-in amplifier.

The thickness of the sample is measured using a differential profilometer based on standard displacement transducers which has been developed in our laboratory. ${ }^{16}$ The output signals from the transducers, each one on each side of the sample, are electronically added and this sum is then amplified. After suitable calibration, this system allows floating thickness measurement, in the sense that none of the sample surfaces is constrained on a flat surface, with a typical precision of a few micrometers (always less than $1 \%$ of the sample thickness). The applied stress is measured with a Force Sensor, Micro Switch FS Series from Honeywell, using the configuration shown in Fig. 2. The sample is held between two hard rubber layers, in order to achieve a smooth fit between the sample and the sample holder.

The control of the $X Y$ system, the trigger for the measurement and the measurements of the detector output signal, applied force and sample thickness are all controlled by software, through a general purpose interface bus communication protocol.

\section{EXPERIMENTAL RESULTS}

\section{A. Monocrystalline silicon wafers}

In order to test the self-calibrating infrared photoelastic (SCIP) method, we have measured the stress variation in a monocrystalline (100) silicon wafer when different known stresses are applied. For each applied stress, the isocromatic

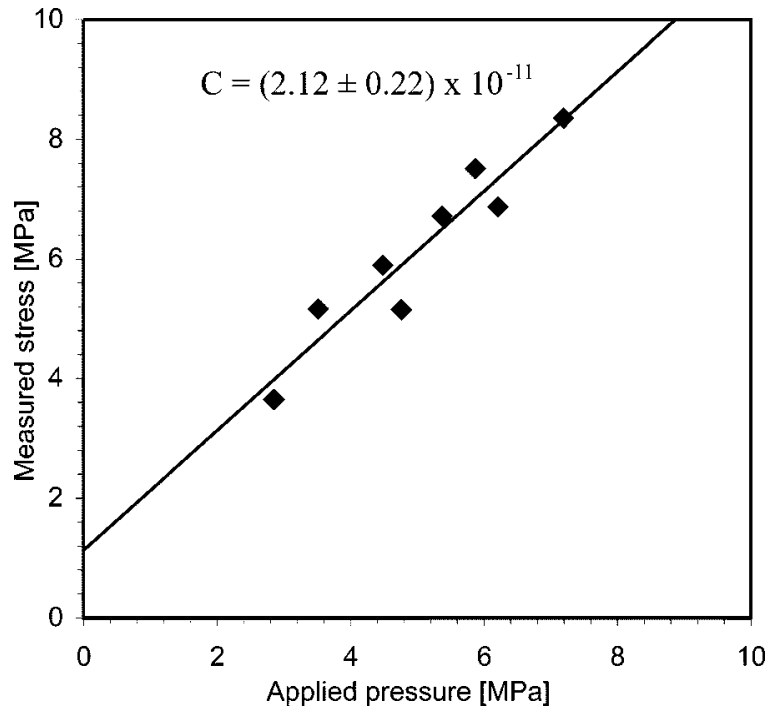

FIG. 3. Measured stress as a function of applied stress on monocrystalline sample.

and isoclinic parameters are determined using Eqs. (5) and (6). Then, Eq. (8) is used to calculate the orientation of the principal components of the stress tensor, $\theta$.

When we apply an external vertical stress $S$, the vertical component of the stress becomes $\sigma_{y}^{\prime}=\sigma_{y}+S$ while both the shear and the horizontal components remain constant $\left(\tau_{x y}^{\prime}\right.$ $=\tau_{x y}$ and $\left.\sigma_{x}^{\prime}=\sigma_{x}\right)$. The new difference between the principal components of the stress tensor can then be written ${ }^{17}$

$$
\begin{aligned}
\left(\sigma_{1}^{\prime}-\sigma_{2}^{\prime}\right)^{2} & =\left(\sigma_{x}-\sigma_{y}-S\right)^{2}+4 \tau_{x y}^{2} \\
& =\left(\sigma_{1}-\sigma_{2}\right)^{2}+S^{2}+2 S\left(\sigma_{1}-\sigma_{2}\right) \cos 2 \theta .
\end{aligned}
$$

If $\theta$ is always small, i.e., the residual stress is aligned with the laboratory reference frame, which is the case for the sample under study, we can write

$$
\left(\sigma_{1}^{\prime}-\sigma_{2}^{\prime}\right)=\left(\sigma_{1}-\sigma_{2}\right)+S .
$$

Using this result and Eq. (4) we can determine the photoelastic coefficient by adjusting it in order to get a unitary slope on the plot $\left(\sigma_{1}^{\prime}-\sigma_{2}^{\prime}\right)$ versus applied stress $S$. Figure 3 shows the variation of the measured stress as a function of the applied stress. The experimental estimation for the photoelastic coefficent for this particular crystal orientation is $(2.12 \pm 0.22) \times 10^{-11} \mathrm{~Pa}^{-1}$.

Since the orientation of the crystal is known, we can use Eq. (7) to compute the expected photoelastic coefficient. We find that the photoelastic coefficent is $C=(2.121 \pm 0.015)$ $\times 10^{-11} \mathrm{~Pa}^{-1}$, which is remarkably consistent with the estimated value.

\section{B. Multicrystalline silicon ribbons}

The SCIP method was used to measure the stress distribution in multicrystalline silicon ribbons produced using a method developed in our laboratory and described elsewhere. ${ }^{4}$ The samples were $100 \mathrm{~mm}$ long and $30 \mathrm{~mm}$ wide. Typical tickness was $300 \mu \mathrm{m}$. Measurements were taken with a $0.3 \mathrm{~mm}$ resolution and with five different external stresses, in the range $0.90-1.90 \mathrm{MPa}$. This stress range 

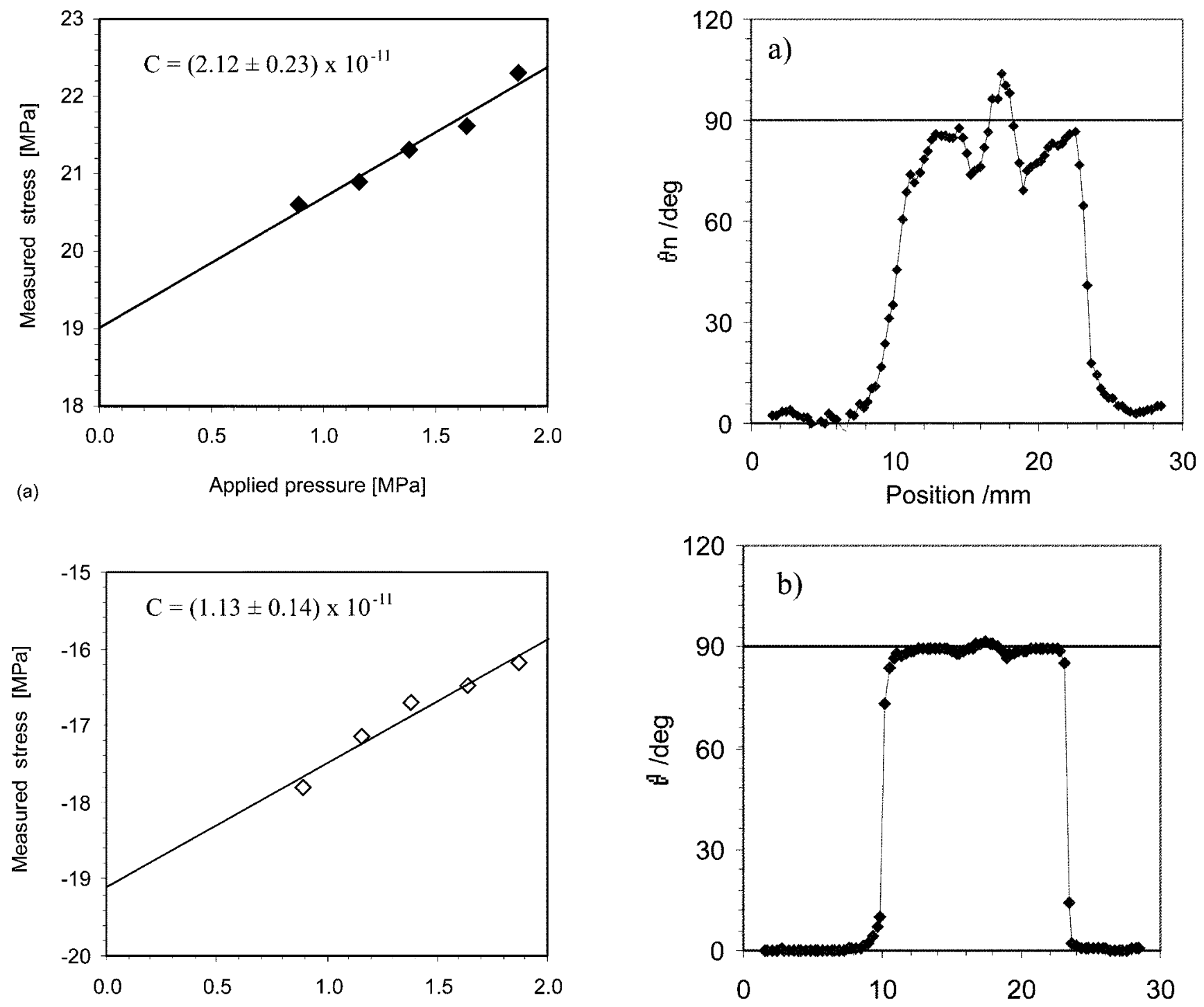

(b)

Applied pressure [MPa]

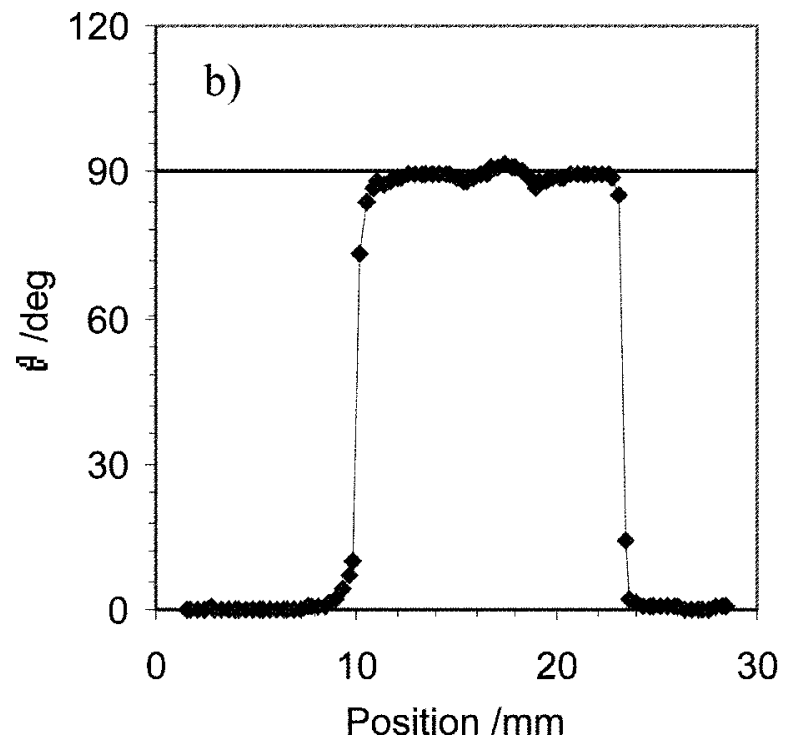

FIG. 4. Measured stress as a function of applied stress on multicrystalline sample for typical points in the (a) edge and (b) central regions of the sample.

was sufficient to determine the residual stress in our samples while avoiding sample breakage. However, this may become a limiting a factor for thinner wafers.

Figure 4 shows the variation of the measured stress with the applied stress on a multicrystalline silicon sample, for typical points in the edge and central regions on the sample. One can see that the variation of the measured stress is quite linear with the applied stress. Furthermore, one ought to notice that in the central region [Fig. 4(b)] the absolute value of the measured stress decreases with increasing the applied stress, which means that that region is under tension. On the other hand, in the outer area [Fig. 4(a)] the measured stress increases with the applied stress, thus suggesting that the regions near the edges of the sample are under compressive residual stress. This result is consistent with thermoelastic models ${ }^{18}$ and stress measurements using shadow moiré interferometry. ${ }^{19}$

Figure 5(a) shows the typical variation of the measured

FIG. 5. (a) Isoclinic parameter and (b) principal orientation of the residual stress. Typical distributions along a line perpendicular to the growth direction of a multicrystalline silicon ribbon.

isoclinic parameter, $\theta_{n}$, along a transversal line (i.e., perpendicular to the growth direction) of the sample. It is clear that the principal orientations of the refractive indices $\left(\theta_{n}\right)$ vary from around $0-10^{\circ}$ in the regions near the edges to around $70-90^{\circ}$ in the central region. Using Eq. (8), we can compute the principal orientation of the residual stress, $\theta$ [Fig. 5(b)].

The principal orientation of the residual stress varies abruptly from values very close to $0^{\circ}$ in the regions near the edges of the ribbon to very close to $90^{\circ}$ in the central region.

According to the thermal stress parabolic model proposed by Gurtler, ${ }^{20}$ silicon ribbons are expected to show $\left|\sigma_{1}\right| \gg\left|\sigma_{2}\right|$ everywhere except in the regions where $\sigma_{1}$ approaches zero, i.e., at $\frac{1}{4}$ and $\frac{3}{4}$ of the length. The variation of $\theta$ can then be interpreted as another evidence for tensile stress in the central region and compressive stress at the edges of the ribbons. Figure 6 shows the variation of the residual stress and the variation of the photoelastic coefficient, determined using the method described in the previous section, along the same transversal line. 

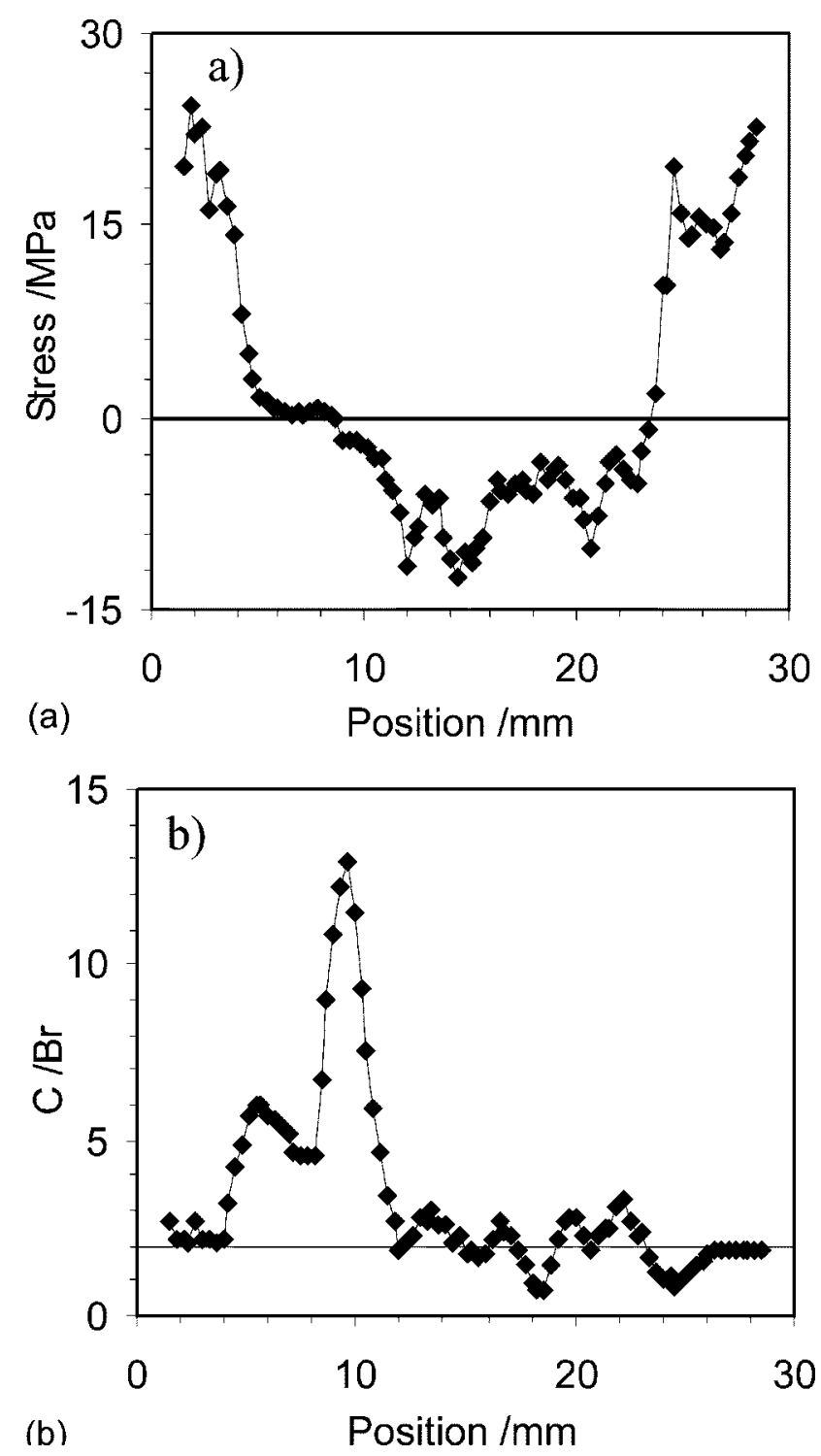

FIG. 6. (a) Residual stress and (b) estimated photoelastic coefficient. Typical distributions along a line perpendicular to the growth direction of a multicrystalline silicon ribbon.

The residual stress distribution shows the basic features of the predictions of the parabolic model, in particular maximum compressive stress is concentrated in the regions near the edges of the ribbon while the central region is under tensile stress. Furthermore, the residual stress becomes neglibible at $\frac{1}{4}$ and $\frac{3}{4}$ of the length of the sample.

The distribution of residual stress is correlated to grain and twin boundaries [Fig. 6(a)]. This result is consistent with stress measurements in EFG silicon ribbons and tri-crystal silicon carried out by Möller and co-workers, ${ }^{21}$ who have associated local high stresses with high density of single slip dislocations that accumulate near grain and twin boundaries.

Since the photoelastic coefficient depends on the crystal orientation, it was expected that it would be constant for each grain, while varying stepwise from grain to grain. Instead, we have observed that the photoelastic coefficient varies within each grain, reaching local maxima in the grain boundaries [cf. Fig. 6(b)]. This result could indicate that the accumulation of dislocations or other defects near grain bound-
TABLE II. Comparison between $\mathrm{x}$-ray diffraction and SCIP stress measurements.

\begin{tabular}{lcccc}
\hline \hline & \multicolumn{2}{c}{ Diffraction } & \multicolumn{2}{c}{ SCIP } \\
\hline Sample & $\begin{array}{c}\sigma_{1} \\
(\mathrm{MPa})\end{array}$ & $\pm \delta \sigma_{1}(\mathrm{MPa})$ & $\begin{array}{c}\sigma_{1} \\
(\mathrm{MPa})\end{array}$ & $\pm \delta \sigma_{1}(\mathrm{MPa})$ \\
\hline A1 & -3.30 & \pm 1.14 & -1.12 & \pm 0.02 \\
A2 & 5.05 & \pm 3.68 & 4.78 & \pm 0.10 \\
B1 & -2.64 & \pm 1.31 & -3.07 & \pm 0.06 \\
\hline \hline
\end{tabular}

aries strongly affects the photoelastic coefficient.

Figure 6(b) also shows unexpectedly large photoelastic coefficients in the region 5-11 mm, a region where $\sigma_{1}-\sigma_{2}$ is small. Furthermore, the applied stress versus measured isocromatic parameter curve has an unusual nonlinear behavior in this region. This suggests that defective material shows an enhanced photoelastic response at low stresses that is only visible where residual stress is small.

Excluding these regions where the photoelastic coefficient is very large, the average photoelastic coefficient is $2.0 \times 10^{-11} \mathrm{~Pa}^{-1}$ and its variation is about $\pm 1.1 \times 10^{-11} \mathrm{~Pa}^{-1}$ which is relatively consistent with the results discussed in Sec. III.

\section{Comparison with x-ray diffraction measurements}

In order to validate the SCIP method, the residual stress of several silicon ribbons was measured by x-ray diffraction using an asymmetric geometry in chosen individual grains. This method will be described in detail elsewhere. ${ }^{22}$ The residual stress, determined from the measured deformation of the unit cell, was shown to be compressive in the outer areas while under tensile stress in the central region.

The results of the x-ray diffraction method cannot be compared directly with the photoelastic measurements since that method only yields the normal components of the stress tensor, not the tangential ones. Thus, we are not able to compute the principal components of the residual stress tensor. However, since we know that the principal orientation of the stress is (almost) everywhere either $0^{\circ}$ or $90^{\circ}$, we can assume that the normal components are the principal components. We can then determine the principal stresses by simply changing from the crystal coordinate system $(a, b, c)$ to the laboratory coordinate system $(x, y, z)$ :

$$
\begin{aligned}
& \sigma_{a}=\sigma_{1} \cos ^{2} \psi, \\
& \sigma_{b}=\sigma_{1} \cos ^{2} \omega, \\
& \sigma_{c}=\sigma_{1} \cos ^{2} \phi,
\end{aligned}
$$

where $\psi, \omega$, and $\phi$ are the orientation angles of the (100) plane around the $x x, y y$, and $z z$ axis, respectively, measured from a lauegram of the sample.

Table II shows the comparison between the measured residual stress obtained by $\mathrm{x}$-ray diffraction and by the SCIP method. It is noticeable that the error bars for the X-ray measurement are quite large. From these results we can conclude 
that the measurement of the residual stress using the X-ray diffraction method is consistent with the SCIP measurements.

\section{ACKNOWLEDGMENTS}

This work was partly supported by SAPIENS and a POCTI scholarship.

${ }^{1}$ J. P. Kalejs, in Silicon Processing for Photovoltaics, edited by C. P. Khattak and K. V. Ravi (Elsevier, Amsterdam, 1987), Vol. II.

${ }^{2}$ A. Eyer, N. Schillinger, S. Schelb, A. Räuber, and J. G. Grabmaier, J. Cryst. Growth 82, 151 (1987).

${ }^{3}$ K. M. Kim, S. Berkman, H. E. Temple, and G. W. Cullen, J. Cryst. Growth 50, 212 (1980).

${ }^{4}$ J. M. Serra and A. M. Vallera, Proceedings of XXI IEEE Photovoltaic Specialists Conference, Orlando, FL, 1990, pp. 615-617.

${ }^{5}$ H. Ghitani and S. Martinuzzi, J. Appl. Phys. 66, 1717 (1989).

${ }^{6}$ B. J. Sloan and J. R. Hauser, J. Appl. Phys. 41, 3504 (1970).

${ }^{7}$ S. R. Lederhandler, J. Appl. Phys. 30, 1631 (1959).

${ }^{8}$ R. O. DeNicola and R. N. Tauber, J. Appl. Phys. 42, 4262 (1971).

${ }^{9}$ D. Chambonnet, R. Gauthier, and P. Pinard, Rev. Sci. Instrum. 57, 2806
(1986).

${ }^{10}$ E. M. Gamarts, P. A. Dobromyslov, V. A. Krylov, S. V. Prisenko, E. A. Jakushenko, and V. I. Safarov, J. Phys. III 5, 1033 (1993).

${ }^{11}$ E. A. Patterson and Z. F. Wang, Strain J. Brit. Soc. Strain Measurement 27, 49 (1991)

${ }^{12}$ T. Zheng and S. J. Danyluk, J. Mater. Res. 17, 36 (2002).

${ }^{13}$ J. F. Nye, Physical Properties of Crystals (Oxford University Press, Oxford, 1972).

${ }^{14}$ H. Liang, Y. Pan, S. Zhao, G. Qin, and K. K. Chin, J. Appl. Phys. 71, 2863 (1992).

${ }^{15}$ K. Ramesh and V. Ganapathy, J. Strain Anal. 31, 423 (1996).

${ }^{16}$ J. Maia Alves, M. C. Brito, J. M. Serra, and A. M. Vallera, Rev. Sci. Instrum. (accepted).

${ }^{17}$ R. G. Budynas, Advanced Strength and Applied Stress Analysis (McGrawHill, New York, 1977).

${ }^{18}$ J. P. Kalejs, B. H. Mackintosh, and T. Surek, J. Cryst. Growth 50, 175 (1980).

${ }^{19}$ Y. Kwon, S. Danyluk, L. Bucciarelli, and J. P. Kalejs, J. Cryst. Growth 82, 221 (1987).

${ }^{20}$ R. W. Gurtler, J. Cryst. Growth 50, 69 (1980).

${ }^{21}$ H. J. Möller, C. Funke, A. Lawerenz, S. Riedel, and M. Werner, Sol. Energy Mater. Sol. Cells 72, 403 (2002).

${ }^{22}$ J. Pereira, M. C. Brito, J. Maia Alves, J. M. Serra, A. M. Vallera, A. Sequeira, and N. Franco (unpublished). 